\title{
Impacto de la epidemia de COVID-19 sobre la actividad de donación y trasplante en el estado de San Luis Potosí
}

\author{
Epidemiologic impact of COVID-19 over donation and \\ transplantation programs in San Luis Potosi, Mexico
}

\author{
Thaydeé G Monsiváis-Santoyo,* Eusermin Gámez-Gómez ${ }^{\ddagger}$ \\ * Directora General, Master en Donación y Trasplantes, Postgrado en Administración y Gerencia en Servicios de Salud. \\ ‡ Director Médico, Master en Donación y Trasplantes, Médico Cirujano. \\ Centro Estatal de Trasplantes de San Luis Potosí (CETRA SLP). México.
}

\section{RESUMEN}

Introducción: En la actualidad no existen reportes de transmisión de COVID-19 relacionados al trasplante de órganos. La actual pandemia por COVID-19 ha generado retos significativos para nuestro sistema de salud en general y para los profesionales de la salud, con repercusiones prominentes en los programas de donación y trasplantes de órganos y tejidos. El objetivo del presente estudio es demostrar el importante impacto que ha dado la pandemia a los programas de donación y trasplantes en el estado de San Luis Potosí. Material y métodos: Se revisaron bases de datos de los archivos de la plataforma del Sistema Informático del Registro Nacional de Trasplantes (SIRNT) de San Luis Potosí durante el periodo del 2015 al 2020. Se compararon las actividades de trasplantes durante los años previos con los realizados en 2020. Resultados: Durante el periodo comprendido, se realizaron 962 trasplantes en el estado de San Luis Potosí, de los cuales 408 fueron de riñón (42.4\%) y 554 de córnea $(57.5 \%)$. Se realizaron 156 trasplantes de origen cadavérico $(37.2 \%)$ y 252 de vivo $(61.7 \%)$. De los trasplantes realizados, 421 se realizaron en mujeres $(43.7 \%)$. Conclusiones: No se encontró diferencia entre donante renal cadavérico y vivo.

Palabras clave: Donación, COVID, coronavirus, trasplantes, riñón.

\section{ABSTRACT}

Introduction: Currently there are no reports of transmission of COVID-19 related to organ transplantation. The current COVID-19 pandemic has created significant challenges for our healthcare system, specially for healthcare professionals, with prominent repercussions on organ and tissue donation and transplant programs observed during the pandemic in the programs of donation and transplant in the country San Luis Potosi. The aim of this study is to prove the impact of the coronavirus pandemic in the program of donation and transplant. Material and methods: The database of the files of the computer System Platform of the National Transplant Registry (SIRNT) with the data of San Luis Potosi was reviewed during the period from 2015 to 2020. The transplant activities during the previous years were compared with those carried out in 2020. Results: During the period covered, 962 transplants were performed in the state of San Luis Potosí, of which 408 were kidney (42.4\%), 554 were corneal (57.5\%). 156 transplants of cadaveric origin (37.2\%) and 252 of living $(61.7 \%)$ were performed. From the transplants performed, 421 were performed in women (43.7\%). Conclusions: There was no difference in the incidence between cadaveric and living kidney donors during the period analyzed.

Keywords: Donation, COVID, coronavirus, transplants, kidney.

Citar como: Monsiváis-Santoyo TG, Gámez-Gómez E. Impacto de la epidemia de COVID-19 sobre la actividad de donación y trasplante en el estado de San Luis Potosí. Rev Mex Traspl. 2021; 10 (1): 12-16. https://dx.doi.org/10.35366/99846 


\section{INTRODUCCIÓN}

A finales del año 2019, se notificaron los primeros casos de neumonías con origen desconocido en Wuhan, perteneciente a la República Popular China. ${ }^{1}$ Después se identificó el agente causal y se le denominó SARSCoV-2 (Severe Acute Respiratory Syndrome Coronavirus 2) cuya manifestación clínica fue nombrada COVID-19 (Coronavirus Disease 2019). El 11 de marzo de 2020, la Organización Mundial de la Salud declaró oficialmente la situación de pandemia. ${ }^{2}$

Ahora son millones ya los casos de infección alcanzados por COVID-19 en todos los continentes. México es uno de los países más afectados, al contar con un gran número de casos reportados, así como de fallecimientos causados por la enfermedad. ${ }^{2}$

No existen reportes de transmisión de COVID-19 relacionados al trasplante de órganos; ${ }^{3}$ sin embargo, múltiples organizaciones, sociedades, colegiados y autoridades en la materia a nivel mundial han emitido recomendaciones para los programas de donación y trasplantes con el objetivo principal de la disminución de riesgo sanitario para los trabajadores de la salud y los pacientes que se encuentran registrados en espera de un trasplante, teniendo en cuenta que éstos constituyen un grupo poblacional de alto riesgo por las comorbilidades que presentan, así como por la inmunosupresión necesaria después del trasplante..$^{4-7}$

La actual pandemia por COVID-19 ha generado retos significativos para nuestro sistema de salud en general y para los profesionales de la salud, con repercusiones prominentes en los programas de donación y trasplantes de órganos y tejidos. Por una parte, la escasez de médicos, enfermeras y técnicos disponibles para mantener la actividad quirúrgica a un ritmo habitual, equipos de protección para el personal de salud con uso preferente en la atención de casos de COVID-19, limitada capacidad de realizar mantenimiento de los potenciales donantes de origen cadavérico ante la escasez de aparatos de ventilación mecánica por la ocupación hospitalaria y necesidades en pacientes COVID-19, rezagado acceso a pruebas para detección de COVID-19, síndrome de burnout en el personal de salud, acondicionamiento de los espacios hospitalarios, diferimiento de traslados de órganos o del equipo quirúrgico por riesgo de contaminación. ${ }^{7}$

Las organizaciones internacionales coinciden en considerar prioridad establecer las condiciones que permitan seguir con el mantenimiento de la activi- dad trasplantadora, la cual NO ES UNA ACTIVIDAD ELECTIVA, ya que la calidad de vida del paciente en espera de un órgano o tejido y la oportunidad del trasplante disminuyen con el transcurso del tiempo. ${ }^{8,9}$

Primordialmente se debe tomar en cuenta el riesgo tanto de los receptores, como de los donantes vivos y del personal de salud que participa en esta actividad respecto a la posibilidad de infección por SARSCoV-2, ${ }^{10}$ por lo que se ha de maximizar la implementación de protocolos para prevenir su transmisión a la población trasplantada, al personal de salud en el ámbito hospitalario y en la comunidad, mediante la optimización de recursos tecnológicos, económicos y humanos. ${ }^{7,11}$ La capacitación constante es punto clave en la toma de decisiones complejas para la asignación óptima de recursos que permita dar respuesta a la demanda creciente de órganos y tejidos para trasplante. $^{12}$

El objetivo del presente estudio es demostrar el importante impacto que ha tenido la pandemia en los programas de donación y trasplantes en el estado de San Luis Potosí.

\section{MATERIAL Y MÉTODOS}

Estudio retrospectivo de la base de datos de pacientes inscritos en la plataforma del Sistema Informático del Registro Nacional de Trasplantes (SIRNT) de las actividades en materia de trasplante del estado de San Luis Potosí durante el periodo 2015 a 2020, incluyendo las actividades realizadas durante los meses de marzo a agosto de cada año.

Se incluyeron en los datos el número total de trasplantes, el número de trasplantes renales y de córnea; el número de trasplantes renales de donante cadavérico (TRDC) y donante vivo (TRDV). Se compararon todos los trasplantes realizados en el periodo de enero a agosto del 2015 al 2019 con los realizados en el mismo periodo en el 2020. Se compararon los porcentajes de cirugía renal y de córnea entre los mismos periodos. Se compararon los trasplantes renales realizados de acuerdo con su origen (TRDC/TRDV), así como el porcentaje de trasplantes realizados por sexo en este periodo.

Se realizaron promedios y desviaciones estándar para variables continuas y promedios para variables categóricas, se evaluó normalidad de los datos con prueba de Shapiro-Wilk, con pruebas de $\chi^{2}$ para porcentajes y $\mathrm{t}$ de Student para variables numéricas en caso de normalidad, también se realizaron pruebas de regresión lineal, con un índice de confianza del 95\%. 


\section{RESULTADOS}

Durante el periodo comprendido, se realizaron 962 trasplantes en el estado de San Luis Potosí, de los cuales 408 fueron de riñón (42.4\%) y 554 de córnea (57.5\%). Se realizaron 156 trasplantes de origen cadavérico (37.2\%) y 252 de vivo (61.7\%), 421 se realizaron en mujeres (43.7\%) (Tabla 1).

En el año 2020 se realizaron 74 trasplantes, de los cuales 31 han sido de riñón y 43 de córnea, que corresponden sólo al $46.1 \%$ del promedio en los mismos meses de los años evaluados $(\mathrm{p}<0.01)$ (Figuras 1 y 2).

En el año 2020 se encontró diferencia significativa en los trasplantes de donante cadavérico y de vivo ( $p$ $<0.01$ ). Sin embargo, en proporción no se encontró diferencia en los trasplantes realizados por sexo entre los diferentes años evaluados, ni entre trasplantes de riñón y córnea en comparación con los otros años.

\section{DISCUSIÓN}

Los programas de trasplantes de órganos requieren una estructura compleja desde el punto de vista médico, logístico y económico. 2,9,13,14

El 30 de marzo, el Consejo de Salubridad General declaró «emergencia sanitaria por causa de fuerza mayor» ${ }^{7,15}$ en consideración a la evolución de la morbilidad y mortalidad relacionadas al SARS-CoV-2, por lo que se desarrollaron acciones como lo fueron las recomendaciones emitidas por parte de las autoridades federales de suspender de forma temporal los programas de donación y trasplantes, pero con la posibilidad de atender casos de urgencia de trasplante 0

Tabla 1: Trasplantes totales.

\begin{tabular}{cccccc}
\hline & $\begin{array}{c}\text { Trasplan- } \\
\text { tes totales } \\
\text { (primeros }\end{array}$ & Trasplantes & Trasplantes & & \\
Año & 8 meses) & de Riñón & de Córnea & TRDC & TRDV \\
\hline 2015 & 110 & 59 & 51 & 21 & 38 \\
2016 & 168 & 67 & 101 & 30 & 37 \\
2017 & 204 & 72 & 132 & 20 & 52 \\
2018 & 241 & 104 & 137 & 44 & 60 \\
2019 & 165 & 75 & 90 & 31 & 44 \\
2020 & 74 & 31 & 43 & 10 & 21 \\
Total & 962 & 408 & 554 & 156 & 252 \\
Promedio & 160.3 & 68 & 92.3 & 26 & 42 \\
\hline
\end{tabular}

TRDC = trasplante renal de donante cadavérico; TRDV = trasplante renal de donante vivo.

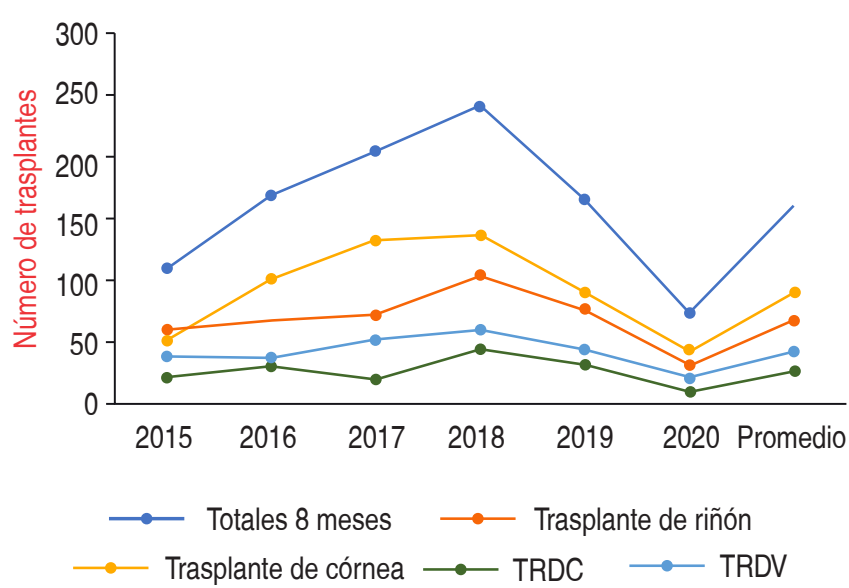

Figura 1: Comparativo de trasplantes realizados durante los primeros ocho meses de cada año, 2015-2020. El máximo en el número total de trasplantes se registró en 2018, mientras el mínimo durante el año 2020. TRDC = trasplante renal de donante cadavérico; TRDV = trasplante renal de donante vivo.

asignaciones prioritarias, como las fallas hepáticas fulminantes, choque cardiogénico o perforación corneal.

Si bien a la fecha no existe evidencia de transmisión de COVID-19 relacionada con el trasplante, es claro que existe un riesgo potencial, por lo que es necesario implementar medidas destinadas a disminuir los riesgos para los receptores del trasplante y profesionales de la salud. ${ }^{5,9,12,16}$ Se considera un alto riesgo de infección por COVID-19 para los equipos de procuración y trasplante, los pacientes y personal de salud, basado en la propagación nosocomial reportada con el SARS-CoV-2.?

España, país líder mundial en trasplante, comunicó que debido al estado nacional de alarma por COVID-19, la actividad de donación y trasplante se redujo de forma sustancial, pasando a una media de 1.1 donantes y 1.8 trasplantes diarios, siendo lo habitual una media de 7.2 donantes y 16.1 trasplantes por día. ${ }^{4}$

La actividad de donación de órganos en México, y específicamente del estado de San Luis Potosí, ha disminuido de manera significativa.

En este estudio se analizaron los primeros ocho meses de cada año para compararlos con los transcurridos durante 2020 hasta la realización de este estudio. Los TRDC son sólo el $46.1 \%$ del promedio de los trasplantes realizados en los mismos meses de los años evaluados. En el caso del trasplante corneal, se ha continuado con mínima diferencia respecto a los años previos; sin embargo, todos los casos registrados durante la pandemia correspondieron a asigna- 
ciones prioritarias con uso de tejido corneal no óptico conservado en resguardo por los bancos de ojos del país, en el caso del trasplante renal, con la publicación del «Plan Nacional de Reactivación de los Programas de Donación y Trasplantes» el 29 de junio, y la implementación de estrategias por CETRA SLP y los establecimientos para el cumplimiento de los lineamientos propuestos, se ha reiniciado localmente el programa de trasplante renal de donante vivo (TRDV).

La necesidad de trasplantar órganos y tejidos no se ha detenido ante la actual contingencia sanitaria, los casos registrados en el SIRNT siguen necesitando de estos procedimientos, ya que sus enfermedades no se detienen, lo que sí se modificó fueron los procedimientos realizados, ya que ahora existe el riesgo de efectuar los procedimientos para receptores y para el personal de salud participante en las donaciones y trasplantes. ${ }^{17}$

El COVID-19 es un factor de exclusión médica para la donación de órganos y tejidos, por lo que se debe descartar de cualquier potencial donante, así como de los pacientes a recibir, ${ }^{7}$ lo que limita los procedimientos y el número de trasplantes a realizar.

En San Luis Potosí se realizaron después de que se emitió el Plan Nacional de Reactivación los cinco primeros trasplantes a nivel nacional (TRDV) sin complicaciones, intensificando las medidas precautorias para evitar contagios; sin embargo, desde marzo que se presentó el primer caso de COVID-19 al mes de agosto no ha habido TRDC, y es que en estos casos

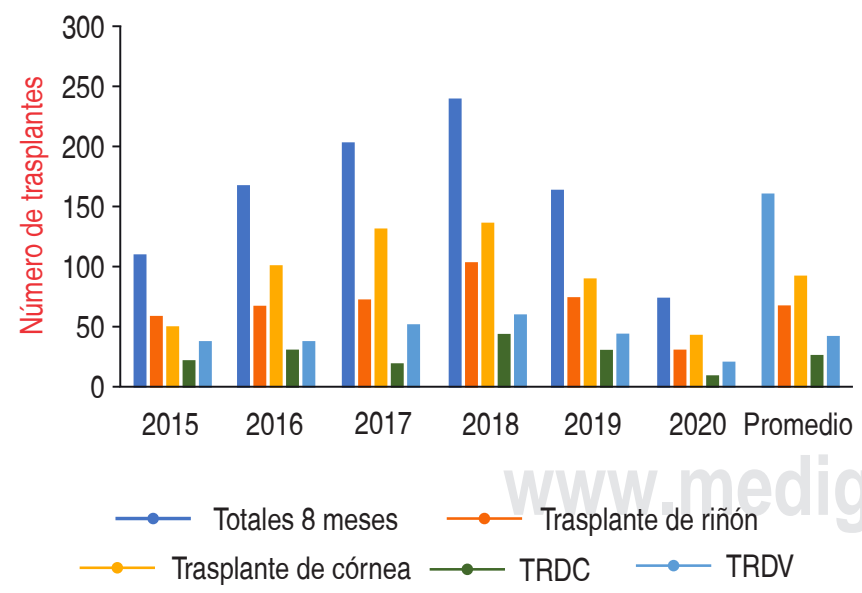

Figura 2: Comparativo de trasplantes realizados en San Luis Potosí durante los primeros ocho meses de cada año, 2015-2020 por tipo de trasplante (y para trasplante renal, por origen del donante).

TRDC = trasplante renal de donante cadavérico; TRDV = trasplante renal de donante vivo. la procuración de órganos y tejidos involucra el contar con recursos humanos, la aceptación familiar para la disposición de órganos y de tejidos, incremento en el tiempo de procuración por la toma de muestra para PCR, disponibilidad de equipos de protección, condiciones hospitalarias para realizar los procesos (áreas libres de COVID), lo cual ha incidido en la negativa de los hospitales que observan un incremento de costos, aunado al impacto económico en los receptores por incorporar dentro de los protocolos exámenes para descartar SARS-CoV-2, dando por resultado la disminución de los procedimientos en general.

\section{CONCLUSIONES}

La pandemia de COVID-19 ha afectado de manera importante el programa de donación y trasplantes. Salvaguardar la salud y calidad de vida de los pacientes implica también facilitar el acceso a los tratamientos y/o cirugías terapéuticas para la insuficiencia orgánica, resulta prioritaria la formación de conocimiento y experiencia que genere certeza y confianza en el reinicio de los programas de trasplante en el país, que ayude a mitigar los efectos secundarios como son numerosos pacientes en espera de ser trasplantados, con deficiente calidad de vida, abandono de las terapias sustitutivas por altos costos, complicaciones que requieren estancia intrahospitalaria y muerte, consecuencia de la pandemia, por lo que no hay mejor forma de enfrentarlo que dar continuidad a los programas de donación y trasplante, generar a partir de la experiencia indicadores clínicos y epidemiológicos apropiados de las acciones de donación y trasplante en relación al COVID-19 que permita la toma de decisiones, mitigar los riesgos y asumir responsabilidad por parte de todos los involucrados en los procesos.

Los trasplantes de donantes fallecidos pueden llevarse a cabo con previsión, analizando cada uno de los casos, tomando en consideración si el hospital cuenta con la capacidad para proporcionar atención postoperatoria adecuada, de la misma forma como se realizó con TRDV.

Ésta era sin precedentes nos plantea un desafío para el personal que se dedica a trasplantes, así como para los pacientes en espera de ser trasplantados por la suspensión y diferimientos de los procedimientos quirúrgicos, también debemos ser conscientes de las situaciones de sufrimiento individual y familiar de nuestros casos por la incertidumbre al no poder ser trasplantados. 


\section{AGRADECIMIENTOS}

\section{Maestro en Ciencias de la Investigación Clínica Dr.} Víctor Román Sánchez Balderas.

\section{REFERENCIAS}

1. Huang C, Wang Y, Li X, Ren L, Zhao J, Hu Y et al. Clinical features of patients infected with 2019 novel coronavirus in Wuhan, China. Lancet. 2020; 395: 497-506. http://dx.doi. org/10.1016/S0140-6736(20)30183-5.

2. World Health Organization. Novel coronavirus situation report 2 [Internet]. 2020. Disponible en: https://www.who.int/docs/ default-source/coronaviruse/situation-reports/20200122-sitrep2-2019-ncov.pdf?sfvrsn $=4 d 5$ bcbca_2

3. López V, Vázquez T, Alonso-Titos J, Cabello M, Alonso A, Beneyto l et al. Recomendaciones en el manejo de la pandemia por coronavirus SARS-CoV-2 (COVID-19) en pacientes con trasplante renal. Nefrologia. 2020; 40 (3): 265-271.

4. Coll E, Gayoso J, Martínez JR, Domínguez B. Informe Casos COVID-19 en pacientes trasplantados. Organización Nacional de Trasplantes. 2020. Disponible en: https://www,seden. org7files7news7INFORME\%20CASOS\%20COVID\%2019\%20 EN\%20PACIENTES\%20TRASPLANTADOS\%20\%2018\%20 MAYOb5f4.pdf

5. Abecassis M, Bridges ND, Clancy CJ, Dew MA, Eldadah B, Englesbe MJ et al. Solid-organ transplantation in older adults: current status and future research. Am J Transplant. 2012; 12 : 2608-2622. http://dx.doi.org/10.1111/j.1600-6143.2012.

6. Roger M, Sierra-Ruiz M, Barrera-Lozano LM. Organ donation and transplantation in the COVID-19 era: should programs really stop? Rev Colomb Cir. 2020; 35: 227-234.

7. Secretaría de Salud-Centro Nacional de Trasplantes. Plan Nacional de Reactivación de los Programas de Donación y Trasplantes. Gobierno de México. 2020. Disponible en: https://www.gob.mx/cenatra/es/documentos/plan-nacionalde-reactivacion-de-los-programas-de-donacion-y-trasplantes246588? state $=$ published

8. Martin R, Sierra-Ruiz M, Barrera-Lozano LM. Donación de órganos y trasplante en la era COVID-19: ¿Realmente se deben parar los programas? Rev Colomb Cir. 2020; 35: 227-234.
9. Daga Ruiz D, Fernández Aguirre C, Segura González F, Carballo Ruiz M. Indicaciones y resultados a largo plazo de los trasplantes de órganos sólidos: Calidad de vida en pacientes trasplantados. Med Intensiva. 2008; 32 (6): 296-303.

10. Martino F, Plebani M, Ronco C. Kidney transplant programmes during the COVID-19 pandemic. Lancet Respir Med. 2020; 8 (5): e39. doi: 10.1016/S2213-2600(20)30182-X.

11. Domínguez-Gil B, Coll E, Ferrer-Fabrega J, Briceño J, Ríos A. Dramatic impact of the COVID-19 outbreak on donation and transplantation activities in Spain. Cir Esp. 2020; 98 (7): 412-414.

12. Documento Técnico. Manejo clínico de pacientes con enfermedad por el nuevo coronavirus (COVID-19). Centro de Coordinación de Alertas y Emergencias Sanitarias. Dirección General de Salud Pública, Calidad e Innovación. Ministerio de Sanidad; 3 de marzo de 2020.

13. Aburto-Morales JS, Romero-Méndez J, Lucio-García CA, Madrigal-Bustamante JA. México ante la epidemia de COVID-19 (SARS-CoV-2) y las recomendaciones al Subsistema Nacional de Donación y Trasplante. Rev Mex Traspl. 2020; 9 (1): 6-14.

14. Garbino J, Crespo S, Aubert JD, Rochat T, Ninet B, Deffernez $C$ et al. A prospective hospital-based study of the clinical impact of non-severe acute respiratory syndrome (Non-SARS)-related human coronavirus infection. Clin Infect Dis. 2006; 43 (8): 10091015. doi: 10.1086/507898.

15. Sánchez-Cedillo A, Cruz-Santiago J, Mariño-Rojas FB, Hernández-Estrada S, García-Ramírez C. Carga de la enfermedad: insuficiencia renal, diálisis-hemodiálisis y trasplante renal en México. Costo de la enfermedad. Rev Mex Traspl. 2020; 9 (1): 15-25.

16. Michaels MG, La Hoz RM, Danziger-Isakov L, Blumberg EA, Kumar D, Green M et al. Coronavirus disease 2019: implications of emerging infections for transplantation. Am J Transplant. 2020; 20 (7): 1768-1772.

17. Villaseñor-Colín C. Principales aspectos quirúrgicos de procuración multiorgánica de donador cadavérico en la contingencia sanitaria por COVID-19. Med Int Mex. 2020; 36 (Suppl: 2): 66-69.

\author{
Correspondencia: \\ Thaydeé G Monsiváis-Santoyo \\ E-mail: thaygrims@gmail.com \\ Eusermin Gámez-Gómez \\ E-mail: eusermin@gmail.com
}

